\title{
Experimental Motion Planning in Airpath Control for HCCI engine
}

\author{
Jonathan Chauvin, Gilles Corde, Nicolas Petit and Pierre Rouchon
}

\begin{abstract}
A motion planning based control strategy is proposed for the airpath control of turbocharged Diesel engines using exhaust gas recirculation (EGR). The considered model uses simple balance equations. The fully actuated dynamics are easily inverted, yielding straightforward open-loop control laws. This approach is complemented by experimentally derived lookup tables to cast the driver's requests into transients between operating points. Estimation of required variables from the intake pressure measurements is addressed and experimental tests are reported on a 4-cylinder engine in Homogeneous Charge Compression Ignition (HCCI) mode. Conclusions stress the possibility of taking into account the non-minimum phase effects of this system by a simple, yet efficient in practice, control law. Observed transients are accurate and fast.
\end{abstract}

\section{INTRODUCTION}

Increasingly stringent pollution standards norms have spurred a broad interest in the reduction of global engine emissions. Lately, two strategies have emerged: aftertreatment and direct combustion emissions reduction. For Diesel engines, equipments required by after-treatment and implementation issues usually carry high cost premiums. An alternative is to use a cleaner combustion mode. Therefore, the Highly Premixed Combustion mode (HPC) - including Homogeneous Charge Compression Ignition (HCCI) - has become of major interest in recent years. It requires the use of high Exhaust Gas Recirculation (EGR) rates. The key idea is that the inert burnt gas (coming from the recirculation of cooled exhaust gas) in the cylinder lower the temperature and dilute the air charge which reduce the emissions of nitrogen oxides. In practice, numerous experimentations brought the proof of significant emission reduction (see [1], [2] for example). Yet, actual vehicle implementation implies frequent transients which reveal to be much more complex than steady state experimentation.

The HCCI combustion mode consists of preparing a highly diluted burnt gas/air/fuel mixture. Simultaneous ignition in the whole combustion chamber is performed and controlled. In that mode, the Burned Gas Rate $(B G R)$ in the intake manifold plays a key role (see Figure 1). Offsets on $B G R$ may cause misfires and additive noises. In the HCCI combustion mode it is very high ( $40 \%$ or more). Accurate control of $B G R$ can be achieved by controlling the whole airpath system: intake and exhaust manifolds, EGR loop and VGT. This is the subject of the paper.

J. Chauvin (corresponding author) is a $\mathrm{PhD}$ Candidate in Mathematics and Control, Centre Automatique et Systèmes, École des Mines de Paris, 60, bd St Michel, 75272 Paris, France chauvin@cas . ensmp. fr

G. Corde is with the Department of Engine Control in Institut Français du Pétrole, 1 et 4 Avenue de Bois Préau, 92852 Rueil Malmaison, France

N. Petit and P. Rouchon are with the Centre Automatique et Systèmes, École des Mines de Paris, 60, bd St Michel, 75272 Paris, France
As studied in [3], [4], the airpath system of a turbocharged Diesel engine features coupled dynamics. The EGR acts as a discharge valve for the turbocharger. Most studies consider the following control setup: both intake and exhaust pressure are closely controlled using EGR valve and Variable Geometry Turbocharger (VGT) (see [5], [6], [7] for example). As presented in [8], [9], [10] multivariable control strategies vastly outperform decentralized mono-variable schemes. Robustness is increased since coupling is taken into account by these multivariable designs. As recalled earlier, HCCI combustion mode requires transients from small to very large $B G R$ rates to be considered. We believe that the previous approaches might not be well adapted to handle such relatively extreme situations.

The contribution of this paper is a control design for the airpath using an explicit feed-forward term. This control law arises from a motion planning open-loop control strategy. Originally, our objective is to control the masses aspirated into the cylinder. We cast it into the control of $B G R$ and pressure in the intake manifold $\left(P_{\text {int }}\right)$. The set points depend on the engine operating conditions: from low load, low pressure and high $B G R$ rate (typically $B G R=40 \%$ and $P_{\text {int }}=1$ bar) to high load, high pressure and no EGR (typically $B G R=0 \%$ and $P_{\text {int }}=2$ bar). We demonstrate the relevance of our approach on such large transients.

The paper is organized as follows. In Section II, we detail the intake manifold model used for our study. It consists of two balance equations. In Section III, we decouple these fully actuated dynamics by a simple motion planning strategy. This yields an open loop control law taking into account the non minimum phase behavior of the system, already underlined in [3]. In Section IV, we design an observer that is required for real implementation: a Luenberger style observer reconstructing poorly known input gains from the intake pressure measurements. Implementation details are given in Section V. Experimental results are reported on a 4 cylinder HCCI engine in Section VI. They stress that, despite important noises on measurements, a control strategy based on a simple model can achieve fast and accurate transients. Conclusions and future directions are given in Section VII.

\section{INTAKE MANIFOLD MODELLING}

Figure 1 shows the flow sheet of the airpath system. Fresh air mixes with the EGR flow into the intake manifold before intake phase. To control the airpath, one can use the Variable Geometry Turbocharger $v_{1}$ (VGT) and the EGR valve $v_{2}$. Modelling can be performed at various geometric and time scales. In this paper, we use mass balances, ideal gas law, and we consider a low time scale resolution $\left(180^{\circ}\right.$ TDC time 


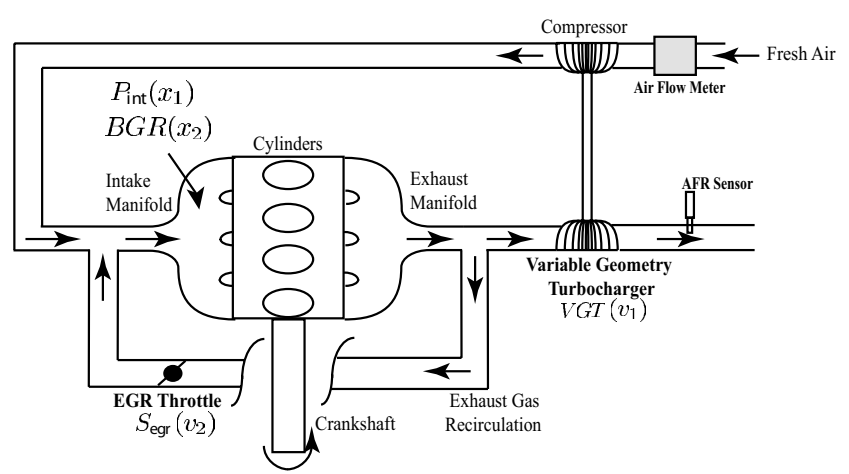

Fig. 1. Engine air path problem.

scale). In particular, high frequency aspiration phenomena are not taken into account.

\section{A. Mass balance in the intake manifold}

Nomenclature is given in Table I. Ideal gas law leads to

$$
P_{\text {int }} V_{\text {int }}=M_{\text {int }} R T_{\text {int }}
$$

The first mass balance writes

$$
\dot{P}_{\text {int }}=\frac{R T_{\text {int }}}{V_{\text {int }}}\left(D_{\text {air }}+D_{\text {egr }}-D_{\text {asp }}\right)
$$

Classically (see [11] for example), we define the aspirated flow as

$$
D_{\text {asp }}=\eta_{\text {vol }}\left(P_{\text {int }}, N_{e}\right) \frac{P_{\text {int }}}{R T_{\text {int }}} V_{\text {cyl }} \frac{N_{e}}{120}
$$

where $V_{\text {cyl }}$ is the cylinder volume. $\eta_{\text {vol }}$ is the volumetric efficiency which is experimentally derived and eventually defined though a look-up table. Values vary with engine operating conditions (mainly intake pressure and engine speed).

On the other hand, the $B G R$ is defined by

$$
B G R \triangleq 1-\frac{M_{\text {air,int }}}{M_{\text {int }}}
$$

The second balance equation is

$$
\frac{d}{d t} B G R=\frac{R T_{\mathrm{int}}}{P_{\mathrm{int}} V_{\mathrm{int}}}\left(D_{\mathrm{egr}}\left(F_{\mathrm{exh}}-B G R\right)-D_{\mathrm{air}} B G R\right)
$$

Composition of the recirculated gases (noted $F_{\text {exh }}$ ) is measured by the Air Fuel Ratio sensor located downstream the turbine.

\section{B. Reference model}

After substitutions, gathering (2) and (3) yield the reference dynamics

$$
\left\{\begin{array}{c}
\dot{P}_{\text {int }}=\gamma_{\text {int }}\left(D_{\text {air }}+D_{\text {egr }}-\eta_{\text {vol }}\left(P_{\text {int }}, N_{e}\right) \Xi_{\text {int }} P_{\text {int }}\right) \\
\frac{d}{d t} B G R=\frac{\gamma_{\text {int }}}{P_{\text {int }}}\left(-\left(D_{\text {air }}+D_{\text {egr }}\right) B G R+F_{\text {exh }} D_{\text {egr }}\right)
\end{array}\right.
$$

where

$$
\gamma_{\text {int }} \triangleq \frac{R T_{\text {int }}}{V_{\text {int }}} \text { and } \Xi_{\text {int }} \triangleq \frac{1}{R T_{\text {int }}} V_{\text {cyl }} \frac{N_{e}}{120}
$$

In a first move, one can expect to directly act upon $D_{\text {air }}$ and $D_{\text {egr }}$. These can be considered as inputs for the reference
TABLE I

NOMENCLATURE.

\begin{tabular}{llll}
\hline Var. & Quantity & Unit & Symb. \\
\hline$P_{\text {int }}$ & Intake pressure & $\mathrm{Pa}$ & $x_{1}$ \\
$B G R$ & Intake burnt gas rate & - & $x_{2}$ \\
$D_{\text {air }}$ & Manifold air flow & $\mathrm{kg} . \mathrm{s}^{-1}$ & $u_{1}$ \\
$D_{\text {egr }}$ & EGR flow & $\mathrm{kg} \cdot \mathrm{s}^{-1}$ & $u_{2}$ \\
$D_{\text {asp }}$ & Air flow into the cylinder & $\mathrm{kg} \cdot \mathrm{s}^{-1}$ & \\
$T_{\text {int }}$ & Intake temperature & $\mathrm{K}$ & \\
$M_{\text {int }}$ & Intake mass & $\mathrm{kg}$ & \\
$M_{\text {air,int }}$ & Intake air mass & $\mathrm{kg}$ & \\
$V_{\text {int }}$ & Intake manifold volume & 1 & \\
$F_{\text {exh }}$ & Fuel Air Ratio & - & \\
$V G T$ & VGT position & $\%$ & $v_{1}$ \\
$S_{\text {egr }}$ & EGR valve effective area & $\%$ & $v_{2}$ \\
$R$ & Gas constant & $\mathrm{J} . \mathrm{kg}$ & \\
$N_{e}$ & Engine Speed & $\mathrm{rpm}$ & \\
$V_{\text {cyl }}$ & Cylinder volume & 1 & \\
$\eta_{\text {vol }}$ & Volumetric efficiency & - & \\
\hline
\end{tabular}

system. Let $x \triangleq\left[\begin{array}{ll}P_{\text {int }} & B G R\end{array}\right]^{T} \in \mathbb{R}^{2}$ be the state and $u \triangleq\left[\begin{array}{ll}D_{\text {air }} & D_{\text {egr }}\end{array}\right]^{T} \in \mathbb{R}^{2}$ the input. Equation (4) leads to the following $2 \times 2$ state space model

$$
\left\{\begin{array}{l}
\dot{x}_{1}=\gamma_{\text {int }}\left(u_{1}+u_{2}-\eta_{\mathrm{vol}}\left(x_{1}, N_{e}\right) \Xi_{\text {int }} x_{1}\right) \\
\dot{x}_{2}=\frac{\gamma_{\text {int }}}{x_{1}}\left(-\left(u_{1}+u_{2}\right) x_{2}+F_{\text {exh }} u_{2}\right)
\end{array}\right.
$$

\section{MOTION PLANNING FOR AIR PATH CONTROL}

Our objective is to control the masses aspirated into the cylinder which is equivalent to find a control solution to the following question: "How to make $P_{\text {int }}$ (i.e. $x_{1}$ ) and $B G R$ (i.e. $x_{2}$ ) reach their respective set points defined by the engine operating conditions (engine speed, load)?". Later, in Section IV, we explain how to estimate these variables. Considered set points are highly varying, especially in HCCI mode. As recalled earlier in the introduction, this mode implies low pressure and high $B G R$ rate at low load (typically $P_{\text {int }}=1$ bar and $B G R>35 \%$ ), while it uses high pressure and no EGR at high load (typically $P_{\text {int }}=3$ bar and $B G R<$ $3 \%$ ). Interaction between air and EGR loops combined with the nonlinear nature of the system between highly varying setpoints makes the system difficult to handle using classical control design methods. Therefore, we now propose a motion planning control strategy which rely on the computation of transient trajectories for the airpath dynamics (5).

\section{A. Motion planning}

System (5) is fully actuated and invertible. Thus, an analytic expression of the input can be derived from the state variable and its first derivatives.

$$
\left\{\begin{array}{c}
u_{1}+u_{2}=\eta_{\mathrm{vol}}\left(x_{1}, N_{e}\right) \Xi_{\mathrm{int}} x_{1}+\frac{1}{\gamma_{\mathrm{int}}} \dot{x}_{1} \\
-x_{2} u_{1}+\left(F_{\mathrm{exh}}-x_{2}\right) u_{2}=\frac{1}{\gamma_{\mathrm{int}}} \dot{x}_{2} x_{1}
\end{array}\right.
$$

This rewrites

$$
\left\{\begin{array}{l}
u_{1}=f_{1}(x, \dot{x}) \\
u_{2}=f_{2}(x, \dot{x})
\end{array}\right.
$$


with

$$
\begin{aligned}
f_{1}(x, \dot{x})=\frac{1}{F_{e x h}} & \left(\frac{F_{\text {exh }}-x_{2}}{\gamma_{\text {int }}} \dot{x}_{1}-\frac{1}{\gamma_{\text {int }}} \dot{x}_{2} x_{1}\right. \\
& \left.+\left(F_{\text {exh }}-x_{2}\right) \eta_{\mathrm{vol}}\left(x_{1}, N_{e}\right) \Xi_{\text {int }} x_{1}\right) \\
f_{2}(x, \dot{x})=\frac{1}{F_{e x h}} & \left(x_{2} \frac{1}{\gamma_{\text {int }}} \dot{x}_{1}+\eta_{\mathrm{vol}}\left(x_{1}, N_{e}\right) \Xi_{\text {int }} x_{2} x_{1}\right. \\
& \left.+\frac{1}{\gamma_{\text {int }}} \dot{x}_{2} x_{1}\right)
\end{aligned}
$$

In these last expressions, $F_{\mathrm{exh}}, \gamma_{\mathrm{int}}, \eta_{\mathrm{vol}}$, and $\Xi_{\text {int }}$ are all given by sensors measurements.

\section{B. Set points}

Given the accelerator position and the engine speed we define one set point. The engine speed, $N_{e}$, is not modelled but directly measured. Accelerator position is turned into a torque control objective yielding an IMEP (Indicated Mean Effective Pressure) set point. The setpoints for the intake pressure and the burnt gas rate are given by experimentally calibrated static maps on the $\left(I M E P^{s p}, N_{e}\right)$ operating range.

$$
\begin{aligned}
& x_{1}^{\mathrm{sp}}=f_{\mathrm{pressure}}\left(I M E P^{\mathrm{sp}}, N_{e}\right) \\
& x_{2}^{\mathrm{sp}}=f_{\mathrm{bgr}}\left(I M E P^{\mathrm{sp}}, N_{e}\right)
\end{aligned}
$$

$I M E P^{\text {sp }}$ is arbitrarily specified by the driver. As IMEP is arbitrarily specified by the driver, $t \mapsto x_{1}^{\mathrm{sp}}(t)$ and $t \mapsto x_{2}^{\mathrm{sp}}(t)$ are neither smooth nor monotonous. These signals must be filtered to correspond to feasible trajectories of (5): filtering gives $x_{1}^{\mathrm{ol}}$ and $x_{2}^{\mathrm{ol}}$. Recalling equation (6), we derive that the unique open-loop control law $\left(u_{1}^{o l}, u_{2}^{o l}\right)$ corresponding to this desired $\left(x_{1}^{o l}, x_{2}^{o l}\right)$ trajectory is defined by

$$
\left\{\begin{array}{l}
u_{1}^{\mathrm{ol}}=f_{1}\left(x_{1}^{\mathrm{ol}}, \dot{x}_{1}^{\mathrm{ol}}, x_{2}^{\mathrm{ol}}, \dot{x}_{2}^{\mathrm{ol}}\right) \\
u_{2}^{\mathrm{ol}}=f_{2}\left(x_{1}^{\mathrm{ol}}, \dot{x}_{1}^{\mathrm{ol}}, x_{2}^{\mathrm{ol}}, \dot{x}_{2}^{\mathrm{ol}}\right)
\end{array}\right.
$$

where $f_{1}$ and $f_{2}$ are defined by (7).

\section{Open loop control}

Formula (8) gives the motion planning laws. The global motion planning control scheme is reported in Figure 2.

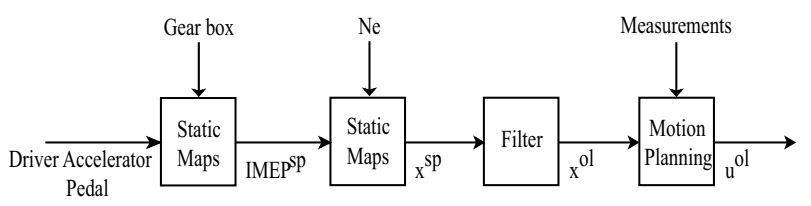

Fig. 2. Motion planning scheme: from torque demand to feedforward generation.

For the sake of illustration, we now briefly present two typical application examples. These results are obtained experimentally in closed loop. Further details about closed loop implementation are given in Section VI where longer horizon results are presented.
1) tip-out: The first scenario is a tip-out (high decrease of torque demand) at $1500 \mathrm{rpm}$ (see Figure 3). Implicitly, it is desired to steer the system from a high load point with no EGR to a low load point with high $B G R$. The proposed open loop control strategy successively opens the EGR valve and then closes the VGT with a significant overshoot. One can notice the resulting decrease of the fresh air flow and simultaneous increase in EGR flow. As expected from a motion planning control strategy, this does provide a soft landing for the state variables $x_{1}$ and $x_{2}$ onto their set points.
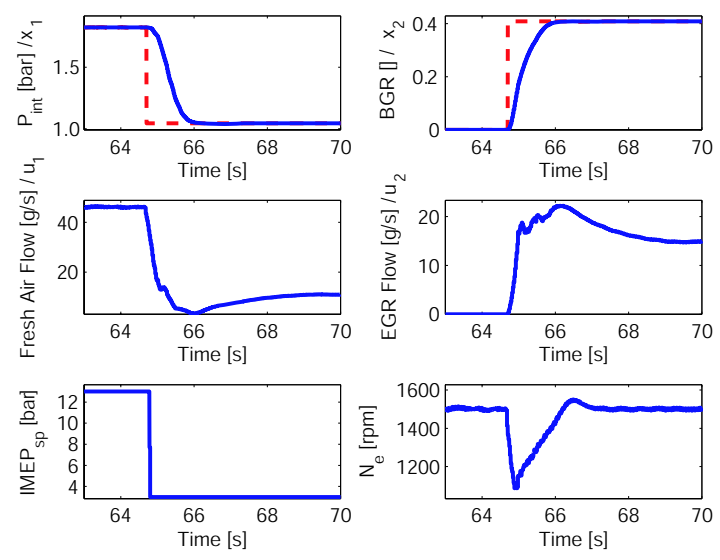

Fig. 3. Experimental results: single tip out from a conventional combustion mode to HCCI combustion mode. IMEP from 13 bar to 3 bar, $B G R$ from 0 to $40.75 \%$, and $P_{\text {int }}$ from 1.825 bar to 1.043 bar at $1500 \mathrm{rpm}$. Dashed red : set point $\left(x^{\mathrm{sp}}\right)$, blue: open loop trajectory $\left(x^{\mathrm{ol}}\right)$.

2) Transient in HCCI combustion mode: The second example is an IMEP transient at $1500 \mathrm{rpm}$ in HCCI combustion mode (see Figure 4). The IMEP of the system starts from 3 bar and reaches 4 bar which implies increasing intake pressure and BGR setpoints. Both operating points are in the HCCI combustion type zone. On contrary to all decentralized controllers, we notice on Figure 4 that our open-loop control take into account the well known non minimum phase behavior of the system as reported in [3]. When the EGR valve opens, the flow increases leading to a pressure rise in the intake manifold. Meanwhile, the exhaust pipe acts as a discharge for the VGT. Its opening lowers the EGR supplied to the turbocharger yielding a significant drop of the exhaust manifold flow. The turbocharger speeds slows down which eventually causes the decrease of the intake manifold pressure. This phenomenon is delayed and slowed down by the turbocharger inertia. Simple ramps and/or steps will fail to let the system reach the desired setpoint. In our case, the model takes into account this complex behavior and therefore the motion planning drives efficiently the system to its setpoint.

\section{AIR PATH OBSERVER}

Before detailing the actual implementation we need to focus on an important estimation issue. Accurate modelling 

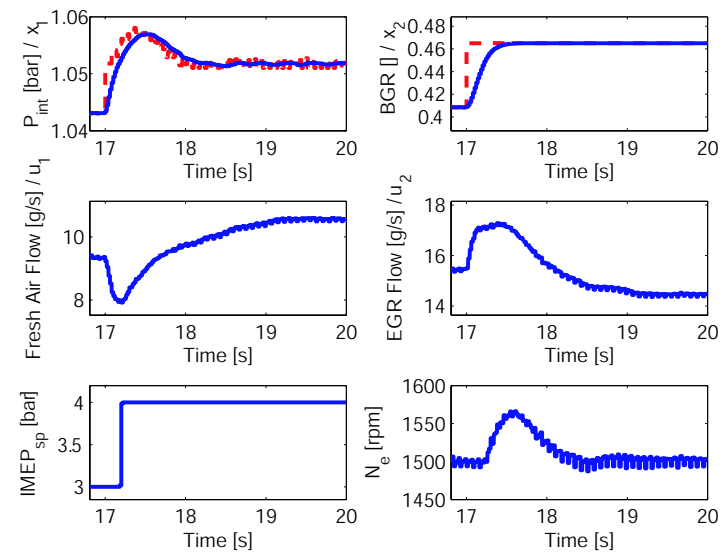

Fig. 4. Transient in the HCCI mode. Experimental results: IMEP from 3 bar to 4 bar, $B G R$ from 40.75 to $43.7 \%, P_{\text {int }}$ from 1.043 bar to 1.105 bar at $1500 \mathrm{rpm}$. Dashed red : set point $\left(x^{\mathrm{sp}}\right)$, blue: open loop trajectory $\left(x^{\mathrm{ol}}\right)$.

of flows through valves can be considered when turbocharger, compressor and cooler models are known and when both exhaust pressure and exhaust temperature are measured. In our case, unfortunately, most of these elements are not available. In practice, while the manifold air flow measurement $D_{\text {air }}$ is relatively accurate, $D_{\text {egr }}$ cannot be measured. We propose an observer to estimate these two required variables along with the $B G R$ in the intake manifold. We note

$$
\left\{\begin{array}{c}
D_{\text {air }}=u_{1}=g_{1}\left(v_{1}\right) \\
D_{\text {egr }}=u_{2}=g_{2}\left(v_{2}\right)
\end{array}\right.
$$

where $g_{1}$ and $g_{2}$ are monotonous mappings of the VGT position $v_{1}$ and the EGR valve effective area $v_{2}$ (these mappings are respectively decreasing and increasing). The EGR is usually reported as a flow through a restriction. In accordance to [11], we choose to model it under the form $g_{2}\left(v_{2}\right) \triangleq \Delta_{2} v_{2}$ where $\Delta_{2}$ is a constant depending on the intake and exhaust pressures and temperatures (and therefore also on the cooling system).

The observer has two interesting features. It reconstructs the EGR flow through $\Delta_{2}$ estimation and estimates the $B G R$. We note $z=\left[\begin{array}{lll}P_{\text {int }} & \Delta_{2} & B G R\end{array}\right]^{T} \in \mathbb{R}^{3}$ the state and $y=P_{\text {int }}$ the measurement. Recalling equation (4), the reference model is

$$
\left\{\begin{array}{l}
\dot{z}_{1}=\gamma_{\text {int }}\left(D_{\text {air }}+z_{2} v_{2}-\eta_{\text {vol }}\left(z_{1}, N_{e}\right) \Xi_{\text {int }} z_{1}\right) \\
\dot{z}_{2}=0 \\
\dot{z}_{3}=\frac{\gamma_{\text {int }}}{z_{1}}\left(-\left(D_{\text {air }}+z_{2} v_{2}\right) z_{3}+F_{\text {exh }} z_{2} v_{2}\right)
\end{array}\right.
$$

\section{A. Observer definition}

We propose the following observer

$$
\left\{\begin{array}{cc}
\dot{\hat{z}}_{1}= & \gamma_{\text {int }}\left(D_{\text {air }}+\hat{z}_{2} v_{2}-\eta_{\mathrm{vol}}\left(y, N_{e}\right) \Xi_{\text {int }} \hat{z}_{1}\right) \\
\dot{\hat{z}}_{2}= & -L_{2}\left(\hat{z}_{1}-y\right) \\
\dot{\hat{z}}_{3}= & \frac{\gamma_{\text {int }}}{y}\left(-\left(D_{\text {air }}+\hat{z}_{2} v_{2}\right) \hat{z}_{3}+F_{\text {exh }} \hat{z}_{2} v_{2}\right)
\end{array}\right.
$$

The (non constant) tuning parameters are

$$
\left\{\begin{array}{l}
L_{1}=\left(l_{1}-\eta_{\mathrm{vol}}\left(y, N_{e}\right)\right) \gamma_{i n t} \Xi_{i n t} \\
L_{2}=l_{2} \gamma_{i n t} v_{2}
\end{array}\right.
$$

where $l_{1}>1$ and $l_{2}>0$ are constant. In particular, we have $L_{1}>0$ and $L_{2}>0$.

$$
\left\{\begin{array}{l}
\dot{\hat{z}}_{1}=\gamma_{i n t}\left(D_{\text {air }}+\hat{z}_{2} v_{2}-l_{1} \Xi_{\text {int }} \hat{z}_{1}\right) \\
\dot{\hat{z}}_{2}=-l_{2} \gamma_{\text {int }} v_{2}\left(\hat{z}_{1}-y\right) \\
\dot{\tilde{z}}_{3}=\frac{\gamma_{\text {int }}}{y}\left(-\left(D_{\text {air }}+\hat{z}_{2} v_{2}\right) \hat{z}_{3}+F_{\text {exh }} \hat{z}_{2} v_{2}\right)
\end{array}\right.
$$

The estimation of both fresh air and EGR flows are

$$
\left\{\begin{array}{l}
\hat{D}_{\text {air }}=D_{\text {air }} \\
\hat{D}_{\text {egr }}=\hat{z}_{2} v_{2}
\end{array}\right.
$$

The state-error is $\tilde{z} \triangleq z-\hat{z}$ and its dynamics writes

$$
\left\{\begin{array}{l}
\dot{\tilde{z}}_{1}=-l_{1} \gamma_{i n t} \Xi_{i n t} \tilde{z}_{1}+\gamma_{i n t} \tilde{z}_{2} v_{2} \\
\dot{\tilde{z}}_{2}=l_{2} \gamma_{i n t} \tilde{z}_{1} v_{2} \\
\dot{\tilde{z}}_{3}=\frac{\gamma_{i n t}}{y}\left(-\left(D_{\text {air }}+\hat{z}_{2} v_{2}\right) \tilde{z}_{3}+\left(F_{\text {exh }}-z_{3}\right) \tilde{z}_{2} v_{2}\right)
\end{array}\right.
$$

\section{B. Convergence analysis}

To investigate convergence of the proposed observer we restrict ourselves to the study around fixed operating point, i.e. $F_{\text {exh }}, T_{i n t}, N_{e}$, and $v_{2}$ are assumed to be constant $\left(\gamma_{i n t}\right.$ and $\Xi_{\text {int }}$ are then constant too). Moreover, we assume that $v_{2}>0$. This last assumption is not restrictive since $v_{2}$ equals 0 implies that the EGR valve is completely closed and that, consequently, the EGR flow and the $B G R$ are equal to 0 .

a) $\left(\tilde{z}_{1}, \tilde{z}_{2}\right)$-dynamics: The errors dynamics (12) is upper-triangular. Indeed, the $\left(\tilde{z}_{1}, \tilde{z}_{2}\right)$-dynamics is independent of $\tilde{z}_{3}$. Moreover, this dynamics is linear

$$
\begin{aligned}
& {\left[\begin{array}{c}
\dot{\tilde{z}}_{1} \\
\dot{\tilde{z}}_{2}
\end{array}\right]=A_{i n t}\left[\begin{array}{l}
\tilde{z}_{1} \\
\tilde{z}_{2}
\end{array}\right]} \\
& \text { where } A_{i n t} \triangleq\left[\begin{array}{cc}
-l_{1} \gamma_{i n t} \Xi_{i n t} & \gamma_{i n t} v_{2} \\
l_{2} \gamma_{i n t} v_{2} & 0
\end{array}\right]
\end{aligned}
$$

$A_{\text {int }}$ is a constant asymptotically stable matrix. This implies that $\tilde{z}_{1}$ and $\tilde{z}_{2}$ are exponentially stable, in other words $\exists\left(\lambda_{\text {int }}, \alpha_{\text {int }, 1}, \alpha_{\text {int }, 2}\right) \in(\mathbb{R} \backslash\{0\})^{3} \quad$ s. t.

$\forall t>0, \quad\left|\tilde{z}_{1}(t)\right| \leq \alpha_{\text {int }, 1} e^{-\lambda_{\text {int } t}}$ and $\left|\tilde{z}_{2}(t)\right| \leq \alpha_{\text {int }, 2} e^{-\lambda_{\text {int }} t}$

b) $\tilde{z}_{3}$-dynamics: On the other hand, the $\tilde{z}_{3}$ dynamics writes

$$
\dot{\tilde{z}}_{3}=-a_{\text {int }}(t) \tilde{z}_{3}+b_{\text {int }}(t) \tilde{z}_{2}
$$

where $a_{i n t} \triangleq \frac{\gamma_{i n t}}{y}\left(D_{\text {air }}+\hat{z}_{2} v_{2}\right)$ and $b_{\text {int }} \triangleq \frac{\gamma_{\text {int }}}{y}\left(F_{\text {exh }}-\right.$ $\left.z_{3}\right) v_{2}$ are bounded strictly positive parameters. For all $t \geq 0$, $0<a_{\mathrm{m}} \leq a_{\text {int }}(t) \leq a_{\mathrm{M}}$ and $0<b_{\mathrm{m}} \leq b_{\text {int }}(t) \leq b_{\mathrm{M}}$. Then,

$$
\dot{\tilde{z}}_{3} \leq-a_{\text {int }}(t) \tilde{z}_{3}+b_{\mathrm{M}} \alpha_{\text {int }, 2} e^{-\lambda_{\text {int }} t}
$$

for $t>0$. Integration leads to

$$
\tilde{z}_{3}(t) \leq-\int_{0}^{t} a_{i n t}(s) \tilde{z}_{3}(s) d s+c_{i n t}^{+}
$$

where $c_{i n t}^{+} \triangleq \tilde{z}_{3}(0)+\frac{b_{\mathrm{M}} \alpha_{\text {int }, 2}}{\lambda_{\text {int }}}$. Gronwall's Lemma (see [12] for example) implies

$$
\tilde{z}_{3}(t) \leq c_{i n t}^{+} e^{-\int_{0}^{t} a_{i n t}(s) d s} \leq c_{i n t}^{+} e^{-a_{\mathrm{m}} t}
$$


Similarly, one can check that there exists a positive real $c_{\text {int }}^{-}$such that $\tilde{z}_{3}(t) \geq-c_{\text {int }}^{-} e^{-a_{\mathrm{m}} t}$. In summary, under the assumption of fixed operating point, the observation error is globally exponentially stable and the following result holds.

Proposition 1: For any fixed operating point, i.e. constant values of $F_{\text {exh }}, T_{i n t}, N_{e}$, and $v_{2}$, assume that $v_{2}>0$, then the state of observer (11) converges exponentially towards the state of system (10).

\section{IMPLEMENTATION}

Both the estimator and the controller described above are tuned in simulation on a high frequency engine model developed in AMESim [13]. This model includes a complete combustion model, balance equations, thermal transfer and gas mixing laws. On the other hand, the test bench used for final validation is a 4 cylinders DI engine with a Variable Geometry Turbocharger (VGT) (see [14] for a complete description) The global control scheme is summarized in Figure 5. The air path observer block (Block (A)) is the

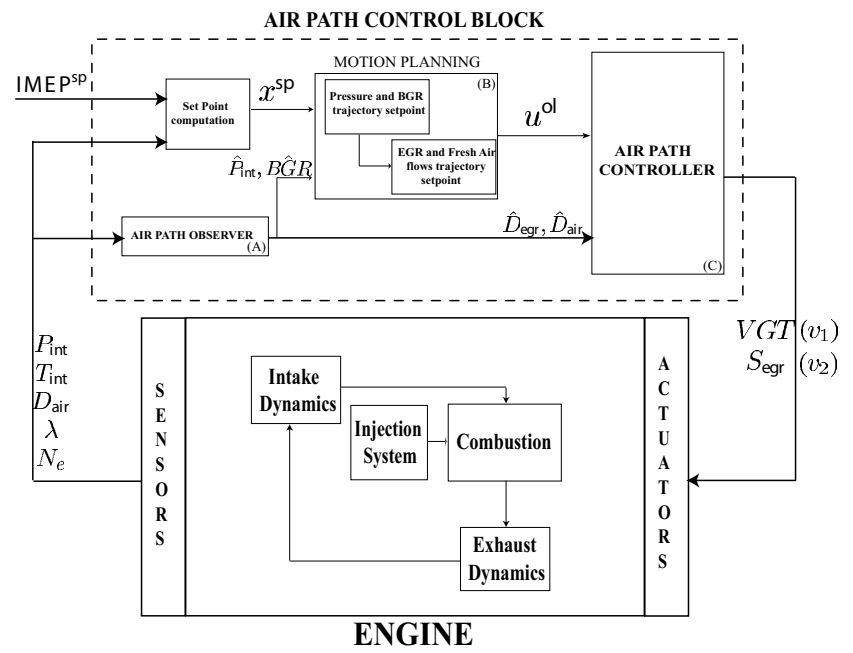

Fig. 5. Control Scheme

implementation of the observer described in Section IV. This block gives an estimation of the $B G R$ and the EGR flow. The motion planning block (Block (B)) is the implementation of the motion planning open loop control strategy described in Section III. Fast PID controllers are added to the structure (Block (C)) to provide further accuracy and robustness. More precisely, a PI controller on the normalized EGR flow $\left(\frac{D_{\text {egrsp }}-\hat{D}_{\text {egr }}}{\hat{D}_{\text {air }}+\hat{D}_{\text {egr }}}\right)$ is used on the EGR valve. Finally, a PID controller on the normalized air flow $\left(\frac{D_{\text {airsp }}-\hat{D}_{\text {air }}}{\hat{D}_{\text {air }}+\hat{D}_{\text {egr }}}\right)$ is used on the VGT, the derivative term being used to compensate the turbocharger inertia. Tuning of these monovariable controllers can be simply addressed through Ziegler-Nichols rules.

\section{EXPERIMENTAL RESULTS}

Experimental results are reported in Figure 6 and Figure 7. Figure 6 represents a decreasing torque demand at constant engine speed (1500 rpm). See Section III-C.1 for complete scenario description. The control reaches the desired values (Intake pressure and $B G R$ ) and $B G R$ is well controlled: fast transient and no overshoot. This is very important in practice since stall are excessive noise are avoided. Figure 7
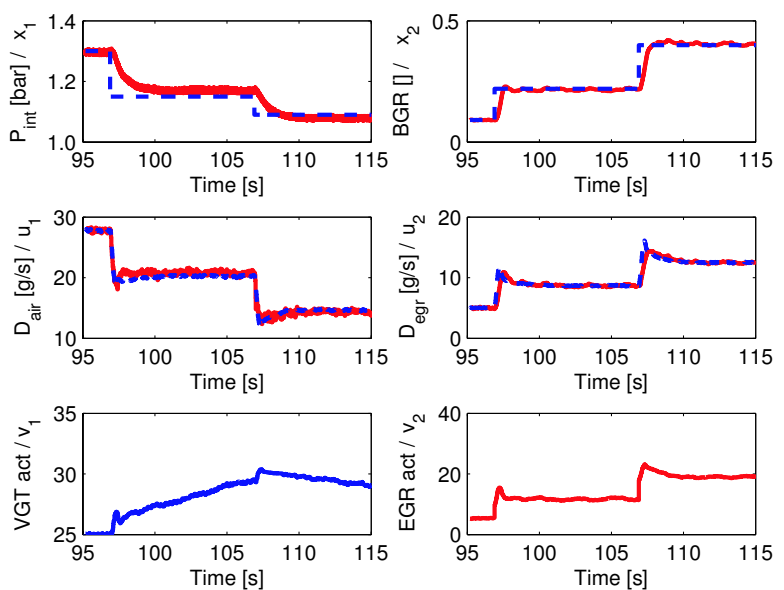

Fig. 6. Experimental results: IMEP from 7 bar (conventional mode) to 6 bar (HCCI mode) to 5 bar (HCCI mode) at $1500 \mathrm{rpm}$. Dashed-blue: set point, red: measurement/estimation.
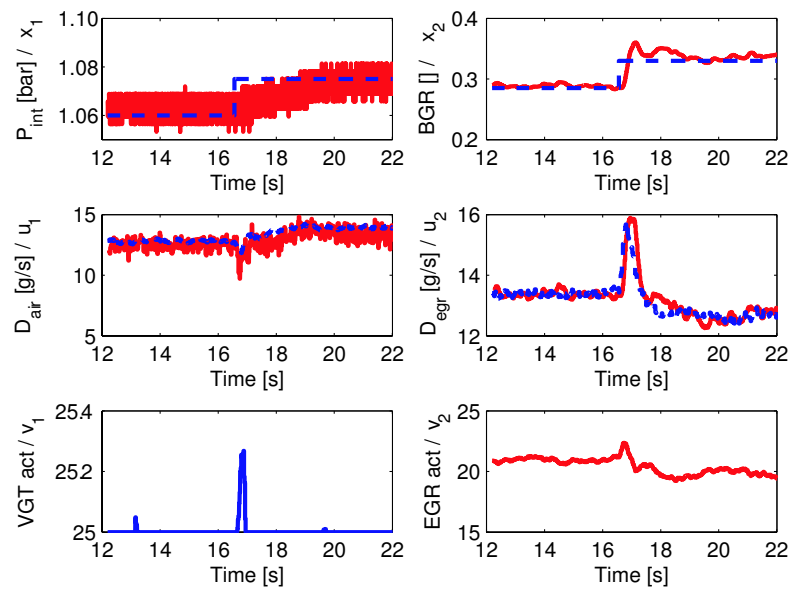

Fig. 7. Transient in the HCCI mode. Experimental results: IMEP from 3 bar (conventional mode) to 4 bar (HCCI mode) at $1500 \mathrm{rpm}$. Dashed-blue: set point, red: measurement/estimation.

represents an increasing torque demand at constant engine speed $(1500 \mathrm{rpm})$ in the HCCI combustion mode. See Section III-C.2 for complete scenario description. Here, we face the non minimum phase behavior of the engine. The open loop control generated by the motion planning applies an inverse response control at the beginning of the transient in order to have a satisfactory response for the $B G R$. Again transients are smooth and present only low oscillations. It is instructive to note that, in this exact same setup, we failed to 
get a decentralized controller preventing experimental issues such as stall and noises and this, due to the overshoot of $B G R$.

\section{CONCLUSION AND FUTURE DIRECTIONS}

The presented work demonstrates the relevance of motion planning in the control of the -coupled- airpath dynamics of turbocharged Diesel engines using Exhaust Gas Recirculation. For the HCCI combustion mode, very large rates of burnt gas need to be considered and we have proven on two realistic test-bench cases that the proposed approach can handle such situations. Besides the results reported here, an exhaustive test campaign is underway to evaluate the performances.

The issue lies in the fact that, for instance, from HCCI to conventional combustion mode, substantial temperature drops are noticed. From now on our model does not address these thermal effects, and will probably not be accurate enough to be used for control purpose. In fact, mismatches in the BGR yielding failure of our control strategy have to be expected. Therefore we are currently working on this point by incorporating a heat balance in our model.

\section{REFERENCES}

[1] J. Kahrstedt, K. Behnk, A. Sommer, and T. Wormbs. Combustion processes to meet future emission standards. In Motortechnische Zeitschrift, pages 1417-1423, 2003.

[2] A. Hultqvist, U. Engdar, B. Johansson, and J. Klingmann. Reacting boundary layers in a homogeneous charge compression ignition (HCCI) engine. In Proc. of SAE Conference, number 2001-01-1032, 2001.

[3] I. Kolmanovsky, A. Stefanopoulou, P. Moraal, and M. van Nieuwstadt. Issues in modelling and control of intake flow in variable geometry turbocharged engines. In Proc of the $18^{\text {th }}$ IFIP Conference on System Modelling and Optimization, 1997.

[4] M. Kao and J. Moskwa. Turbocharged Diesel engine modelling for nonlinear engine control and estimation. ASME Journal of Dynamic Systems, Measurements and Control, 117, 1995.

[5] M Janković and I. Kolmanovsky. Constructive Lyapounov control design for turbocharged Diesel engines. IEEE Transactions on Control Systems Technology, 8:288-299, 2000.

[6] M Janković and I. Kolmanovsky. Robust nonlinear controller for turbocharged Diesel engine. In Proc. of the American Control Conference, 1998.

[7] M. Ammann, N. Fekete, L. Guzzella, and A. Glattfelder. Model-based control of the VGT and EGR in a turbocharged common-rail Diesel engine: theory and passenger car implementation. In Proc. of SAE Conference, number 2003-01-0357, 2003.

[8] M. van Nieuwstadt, P. Moraal, I. Kolmanovsky, A. Stefanopoulou, P. Wood, and M. Criddle. Decentralized and multivariable designs for EGR-VGT control of Diesel engine. In Proc of the $2^{\text {nd }}$ IFAC Workshop on Advances in Automotive Control, 1998.

[9] M. van Nieuwstadt, I. Kolmanovsky, P. Moraal, A. Stefanopoulou, and M. Janković. Experimental comparison of EGR-VGT control schemes for a high speed Diesel engine. Control System Magazine, 20:63-79, 2000.

[10] A. Stefanopoulou, I. Kolmanovsky, and J. Freudenberg. Control of variable geometry turbocharged Diesel engines for reducted emissions. IEEE Transactions on Control Systems Technology, 8:733-745, 2000.

[11] J. Heywood. Internal Combustion Engine Fundamentals. McGrawHill, Inc, 1988.

[12] H. Khalil. Nonlinear Systems. Prentice-Hall, Inc., 1992.

[13] IMAGINE. AMESim user manual, http://www.amesim.com, 2004.

[14] A. Albrecht, J. Chauvin, S. Potteau, and G. Corde. Design of real-time torque balancing control for highly premixed combustion engine using a 1d Diesel engine model. In Proc. of the IAV Conference "Engine process simulation and supercharging”, 2005. 